\title{
A Comparison of Changes in the Fatty Acid Profile of Human Milk of Spanish Lactating Women during the First Month of Lactation Using Gas Chromatography-Mass Spectrometry. A Comparison with Infant Formulas
}

\author{
Silvia Sánchez-Hernández ${ }^{1,2, *}$, Adelaida Esteban-Muñoz ${ }^{1,2}$, Rafael Giménez-Martínez ${ }^{1,3}$, \\ María José Aguilar-Cordero ${ }^{4}$, Beatriz Miralles-Buraglia ${ }^{5}$ and Manuel Olalla-Herrera ${ }^{1,3}$ \\ 1 Department of Nutrition and Bromatology, University of Granada, 18071 Granada, Spain; \\ aidaem@ugr.es (A.E.-M.); rafaelg@ugr.es (R.G.-M.); olalla@ugr.es (M.O.-H.) \\ 2 Programme in Nutrition and Food Science, University of Granada, 18071 Granada, Spain \\ 3 Instituto de Investigación Biosanitaria, 18071 Granada, Spain \\ 4 Department de Nursing, University of Granada, 18071 Granada, Spain; mariajaguilar@telefonica.net \\ 5 Institute of Food Science Research (CIAL), CSIC-UAM, 28049 Madrid, Spain; beatriz.miralles@csic.es \\ * Correspondence: silsanchez@ugr.es
}

Received: 13 November 2019; Accepted: 12 December 2019; Published: 14 December 2019

\begin{abstract}
Breastfeeding is the ideal way to provide infants with the nutrients they need for healthy growth and development. Milk composition changes throughout lactation, and fat is one of the most variable nutrients in human milk. The aim of this study was to determine the main differences between the fatty acid (FA) profile of human milk samples (colostrum, transitional, and mature milk group) and infant formulas. Human milk samples were provided by lactating women from Granada. Moreover, different commercial infant formulas were analyzed. FAs were determined using gas chromatography coupled with mass spectrometry. According to the results, oleic acid was the predominant monounsaturated fatty acid (41.93\% in human milk and $43.53 \%$ in infant formulas), while palmitic acid was the most representative saturated fatty acid $(20.88 \%$ in human milk and $23.09 \%$ in infant formulas). Significant differences were found between human milk groups and infant formulas, mainly in long-chain polyunsaturated FAs (LC-PUFAs). The content of araquidonic acid (AA) and docoxahexaenoic acid (DHA) was higher in human milk $(0.51 \%$ and $0.39 \%$, respectively) than in infant formulas ( $0.31 \%$ and $0.22 \%$, respectively). Linoleic acid (LA) percentage $(15.31 \%)$ in infant formulas was similar to that found in human milk (14.6\%). However, $\alpha$-linolenic acid (ALA) values were also much higher in infant formulas than in human milk (1.64\% and $0.42 \%$, respectively).
\end{abstract}

Keywords: fatty acids; human milk; infant formula; GC-MS/MS; LC-PUFA

\section{Introduction}

Breastfeeding is the ideal way to provide young children with the nutrients they need for healthy growth and development [1]. The World Health Organization (WHO) and the United Nations International Emergency Fund for Children (UNICEF) adopted measures in 2002 to promote global health. As a part of this, the global strategy for optimal food use in infants and young children recommended breastfeeding from the first hour of life, continuing with exclusive breastfeeding during the first 6 months of life and further breastfeeding up to 2 years of age which is supplemented with other foods [2,3]. Data published in 2016 by UNICEF indicates that, overall, only $43 \%$ ( 2 out of 5 ) of children continue to receive exclusive breastfeeding at 6 months of age [4]. This is because some mothers cannot or choose not to breastfeed and 
instead use infant formulas (IF) as a substitute. IF are manufactured foodstuffs for feeding newborns and babies that attempts to match, as much as possible, the composition of human milk (HM), especially the lipid profile [5]. Lipids are the largest source of energy in human milk. Triacylglycerols (TAGs) represent 98-99\% of total fats and their properties are determined by the length of and degree of unsaturation of fatty acids (FAs) esterified to the glycerol backbone [6]. Milk composition changes throughout lactation, and fat is one of the most variable nutrients in human milk. Lipid content changes according to the stage of lactation and time of day, and during feeding. Whilst in human milk the composition of fatty acids is dynamic and modulated by maternal diet, infant formulas have a much less complex composition than human milk fat [6-8]. This can provide challenges in attempting to ensure normal or typical fatty acid intake in breastfed infants and in establishing fatty acid targets when developing infant formulas [8].

Human milk contains the parent essential fatty acids (EFA) linoleic acid (LA, C18:2 n-6) and $\alpha$-linolenic acid (ALA, C18:3 n-3), and n-3/n- 6 very long-chain polyunsaturated fatty acids (LC-PUFA) [9]. A balanced amount of these fatty acids is required for normal maturation and functioning of the nervous system [10]. These fatty acids are also associated with the development of allergic diseases and inflammatory responses [11]. They regulate growth, alongside visual, cognitive, and motor development during the first year of life [12-15].

LC-PUFA are present in human milk in concentrations greater than in other commercially available milks [16]. This is biologically relevant since the two major LC-PUFA are arachidonic acid (AA, C20:4 $\mathrm{n}-6$ ) and docosahexaenoic acid (DHA, C22:6 n-3). These accumulate in the fetal retina and brain during the last trimester of pregnancy and the early period of postnatal development when milk represents the only source of fat $[13,16]$.

Infant formulas provide all of these essential nutrients for adequate growth and development. These nutrients include the LA and ALA, as required by regulatory agencies [17], although the addition of LC-PUFA as AA and DHA is not mandated [18].

In recent years, variability of the fatty acid profile in human milk and in infant feeding has become very important. For this, the aim of the present study was:

(1) To determine the main differences between the fatty acid profile of human milk samples at three stages of lactation (colostrum, transitional, and mature milk group) in women from Granada and compare the fatty acid profile of human milk with different commercially available infant formulas in Spain. This will provide new fatty acid data through use of novel analytical techniques.

(2) To study the relationship between different milk samples according to their FA composition and determine which fatty acids are mostly responsible for the differences found between human milk samples and infant formulas.

\section{Materials and Methods}

\subsection{Chemicals and Reagents}

Fatty acid methyl esters (Supelco 35 Component FAME Mix) were obtained from Sigma-Aldrich SL (Madrid, Spain). N-hexane, isopropanol, anhydrous sodium sulfate, undecanoic acid (C11:0), methyl acetate, sodium methoxide, methanol, oxalic acid, and diethyl ether were acquired from Panreac Química SL (Panreac AppliChem, Barcelona, Spain) and Sigma-Aldrich (Merck, Munich, Germany). All reagents were of analytical grade.

\subsection{Subject}

The present research was carried out in the obstetrics department of one of the six regional hospitals of Andalusia "Hospital Universitario Virgen de las Nieves". Of all of the participants who formed part of this study, the three samples of human milk (colostrum, transitional, and mature milk) were donated by only thirteen lactating mothers due to the complexity of obtaining these samples. Details of the study were explained to all mothers who voluntarily gave written consent to participate. The characteristics of the study sample were as follows: women aged $18-40$, who had given birth 
to healthy babies in the "Hospital Universitario Virgen de las Nieves". This also constituted the inclusion criteria.

Ethical approval for this study was provided by the "Hospital Virgen de las Nieves" Ethics and Scientific Committee and the trial was registered at ClinicalTrials.gov.

The mean age of participating mothers was $32 \pm 4.7$ years, of which eight reported this being their first lactation/child (N1, N2, N3, N5, N6, N10, N11, and N12).

The main characteristics of sampled mothers are shown in Table 1.

Table 1. Characteristics of the sampled mothers.

\begin{tabular}{ccccc}
\hline & Mean & SD & Minimum & Maximum \\
\hline Age (years) & 32 & 4.7 & 26 & 40 \\
Height $(\mathrm{cm})$ & 163.6 & 7.00 & 150.0 & 172.0 \\
Weight $(\mathrm{kg})$ & 69.6 & 13.50 & 47.4 & 90.8 \\
BMI $\left(\mathrm{kg} / \mathrm{cm}^{2}\right)$ & 26.2 & 5.33 & 18.4 & 33.4 \\
Birth weight $(\mathrm{kg})$ & 2.9 & 0.79 & 1.2 & 3.8 \\
Parity & 1.6 & 0.92 & 1 & 3 \\
\hline
\end{tabular}

BMI: body mass index; SD: standard deviation.

\subsection{Milk Samples}

Thirty-eight milk samples were categorized according to the length of time post-partum. Samples obtained between the 1st and 5th day post-delivery were assigned to the colostrum group $(n=13$, $1.75 \pm 0.53$ days) with the Marmet manual extraction technique being used for delivery [19]; samples obtained between the 6th and 15th day post-delivery were assigned to the transitional group $(n=13$, $12.83 \pm 4.43$ days); and samples obtained after the 15th day post-delivery were assigned to the mature milk group ( $\mathrm{n}=12,24.75 \pm 9.77$ days). For both of the latter groups, milk extraction was achieved by means of a mechanical breast pump (Medela ${ }^{\circledR}$, Medela, Switzerland) following the manufacturer's instructions. Milk from each breast was obtained at both the beginning and end of each feed.

All human milk samples collected from study participants were aliquoted and immediately stored at $-70{ }^{\circ} \mathrm{C}$ until extraction.

In addition to the human milk samples, seven different initiation formulas (0-6 months) for full-term infants were also analyzed. These are the most commonly consumed infant formulas and include brands such as Nestlé, Combiotik, Blemil, Nutribén, and Almirón, and were purchased in different commercial and agricultural areas of the market. They were randomly coded as IF 1-7 (Table S1). All samples were analyzed in triplicate.

\subsection{Total Lipid Content and Fat Extraction}

Infant formulas were reconstituted in water following the manufacturer's instructions.

The human milk samples and infant formulas were extracted with a mixture of solvents according to the method of Hara and Radin [20].

First, $500 \mu \mathrm{L}$ of milk was mixed with $1.8 \mathrm{~mL}$ of n-hexane, isopropanol $(3: 2, v / v)$, then and homogenized. Next, $1.2 \mathrm{~mL}$ of aqueous sodium sulfate was added and centrifuged to separate the layers. The organic layer was evaporated using nitrogen (N2). The aqueous phase was re-extracted, and the lipid recovered and stored at $-18{ }^{\circ} \mathrm{C}$ in $n$-hexane: isopropanol $(4: 1, v / v)$ until the fatty acid methyl esters (FAME) were prepared.

\subsection{Preparation of Methyl Esters for Gas Chromatographic Analysis}

Fatty acid methyl esters (FAME) were obtained after following the base-catalyzed transesterification method described by Christie WW. [21]. The previously evaporated sample was dissolved in $0.5 \mathrm{~mL}$ of n-hexane. $40 \mu \mathrm{L}$ of methyl acetate and $80 \mu \mathrm{L}$ of sodium methoxide in methanol $(0.5 \mathrm{M})$ were added. The solution was mixed for $30 \mathrm{~s}$ and left for $15 \mathrm{~min}$ at room temperature, at which time the reaction was stopped by adding $30 \mu \mathrm{L}$ of a saturated solution of oxalic acid in diethyl ether. After a brief agitation, 
the mixture was centrifuged at $1500 \times \mathrm{g}$ for $2 \mathrm{~min}$ and the supernatant, containing FAME, was collected into chromatography vials.

\subsection{Chromatographic and Mass Operating Conditions}

Determination of fatty acids was conducted using a mass spectrometer with tandem quadrupole model QUATTRO micro GC (WATERS, Milford, MA, USA), equipped with a split/splitless injector. A Flame Ionization Detector (FID) type detector with ionization mode EI+ at $300{ }^{\circ} \mathrm{C}$ was used, measuring a mass range of 45 to 450 and a 35 min chromatogram, and a capillary column with a length of $30.0 \mathrm{~m}$ and a diameter of $250 \mu \mathrm{m}$. The temperature of the initial oven was $100^{\circ} \mathrm{C}$ with a maximum of $350{ }^{\circ} \mathrm{C}$. The injection volume was $1 \mu \mathrm{L}$ and the split ratio was set at 10.0 and split flow $10.0 \mathrm{~mL} / \mathrm{min}$.

All data was collected using MassLynx V4.1 software (Waters Inc., 2010, Milford, MA, USA). Peaks were identified by comparing retention times with standard mixtures. Fatty acids were quantified by comparing the peak area of each compound with that of the standard.

\subsection{Analytical Validation}

Validation was carried out by studying the parameters of linearity, linear range, limit of detection (LOD), and limit of quantification (LOQ), following the guidelines for the validation of analytical methods (AOAC, 2012). Linearity in all fatty acids was achieved in their dynamic range of between 250-1000 ppm. A total of 35 fatty acids were identified and quantified in the different milk samples.

This is a highly sensitive and precise analytical method based on mass spectrometry combined with high resolution separation methods.

\subsection{Statistical Analyses}

The homogeneity of variance was assessed using the Levene test and normality of data distribution of the samples was examined with the Shapiro-Wilk test.

Results of individual FA content of the different human milk samples and infant formulas were analyzed using one-way ANOVA followed by the Tukey test in order to compare significant variations between means $(p<0.05)$. Moreover, in order to verify the capacity of the FA analysis as a tool for human milk characterization, a multivariate discriminant analysis was performed. Graphical representation of this analysis allows the similarity of samples to be assessed according to their FA composition.

The significance level was set at $5 \%(p<0.05)$ in all tests. SPSS 15.0 for Windows (IBM SPSS Inc., Chicago, IL, USA) was used for data analyses.

In order to complete the examination of the fatty acid profile of human milk and infant formulas, the results obtained were subjected to discriminant analysis. This analysis is aimed at supporting the interpretation of complex multivariate data. It considers all observations as a single group with the aim of uncovering the variables with the greatest influence, so that observations can be grouped and predictive groups formed. The data obtained were expressed as means \pm standard deviations (SD).

\section{Results}

\subsection{Analytical Validation}

A chromatogram of the standards of these fatty acids over a period of $35 \mathrm{~min}$ is shown in Figure 1.

Table 2 shows the linear range, retention time, adjusted linear equations, correlation coefficients, detection limits, and quantification limits of the standards.

A favorable correlation between the experimental data and the theoretical values was obtained with good linearity in the ranges evaluated and correlation coefficients (R2) greater than 0.998 .

The highest LOD and LOQ corresponded to $\alpha$-linolenic acid (C18:3 n-3) (1.8403 and 6.1344) and the lowest to arachidonic acid (C20:4 n-6) (0.1345 and 0.4482). These LOD and LOQ were compared with values previously reported by other authors for fatty acids using GC-MS/MS methods. 


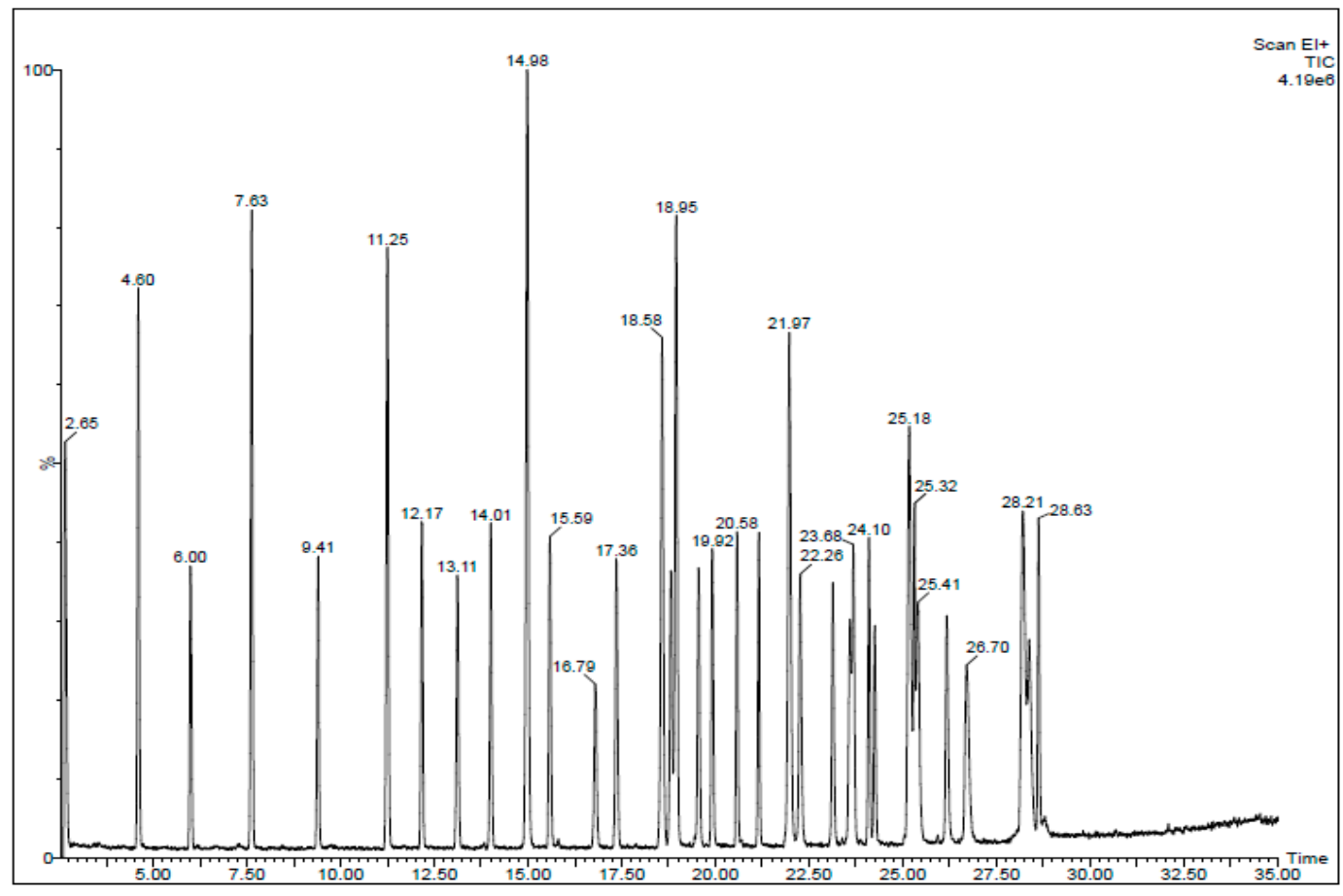

Figure 1. Chromatogram of a 35 component Fatty Acid Methyl Esters (FAME) mix of the standard. 
Table 2. Quality parameters for the chromatographic determination of fatty acid standards.

\begin{tabular}{|c|c|c|c|c|c|c|c|}
\hline Fatty Acids & Linear Range (ppm) & $t_{r} \pm S D$ & Linear Equation & $R$ & $R^{2}$ & LOD (ppm) & LOQ (ppm) \\
\hline Caprylic acid (C8:0) & $250-1000$ & $2.647 \pm 0.006$ & $y=271.7940 x+813.2345$ & 0.9999 & 0.9997 & 0.3362 & 1.1208 \\
\hline Capric acid (C10:0) & $250-1000$ & $4.597 \pm 0.006$ & $y=362.0136 x-8269.8440$ & 0.9999 & 0.9997 & 0.3223 & 1.0743 \\
\hline Undecanoic acid (C11:0) & $250-1000$ & $6.000 \pm 0.000$ & $y=183.0014 x-4785.7425$ & 0.9999 & 0.9998 & 0.4375 & 1.4583 \\
\hline Lauric acid (C12:0) & $250-1000$ & $7.623 \pm 0.006$ & $y=403.2163 x-11475.9145$ & 0.9998 & 0.9996 & 0.2929 & 0.9765 \\
\hline Tridecanoic acid (C13:0) & $250-1000$ & $9.400 \pm 0.010$ & $y=197.6763 x-6117.3745$ & 0.9999 & 0.9999 & 0.5236 & 1.7452 \\
\hline Myristic acid (C14:0) & $250-1000$ & $11.247 \pm 0.006$ & $y=414,5987 x-8987,9605$ & 0.9994 & 0.9988 & 0.1620 & 0.5400 \\
\hline Myristoleic acid (C14:1) & $250-1000$ & $12.167 \pm 0.006$ & $y=217,1785 x-9140,8480$ & 0.9993 & 0.9987 & 0.1514 & 0.5046 \\
\hline Pentadecanoic acid (C15:0) & $250-1000$ & $13.117 \pm 0.012$ & $y=211,0244 x-6724,5895$ & 0.9996 & 0.9993 & 0.2058 & 0.6862 \\
\hline Cis-10-pentadecenoic acid (C15:1) & $250-1000$ & $14.010 \pm 0.010$ & $y=221,6763 x-9651,3250$ & 0.9999 & 0.9998 & 0.2058 & 0.6862 \\
\hline Palmitic acid (C16:0) & $250-1000$ & $14.980 \pm 0.010$ & $y=663,2947 x-24193,3435$ & 0.9999 & 0.9998 & 0.3793 & 1.2642 \\
\hline Palmitoleic acid (C16:1 n-9 Z) & $250-1000$ & $15.587 \pm 0.006$ & $y=227,0054 x-8233,3830$ & 0.9996 & 0.9991 & 0.1901 & 0.6337 \\
\hline Margaric acid (C17:0) & $250-1000$ & $16.793 \pm 0.006$ & $y=147,9136 x-5182,7135$ & 1.0000 & 1.0000 & 0.9664 & 3.2212 \\
\hline Cis-10-heptadecenoic acid (C17:1) & $250-1000$ & $17.357 \pm 0.006$ & $y=229,9246 x-10188,1020$ & 0.9999 & 0.9998 & 0.4456 & 1.4855 \\
\hline Stearic acid (C18:0) & $250-1000$ & $18.577 \pm 0.006$ & $y=464,1352 x-13723,7815$ & 0.9997 & 0.9995 & 0.2437 & 0.8123 \\
\hline Elaidic acid (C18:1 n-9 E) & $250-1000$ & $18.817 \pm 0.006$ & $y=158.1385 x-2278.2970$ & 1.0000 & 0.9999 & 0.6563 & 2.1877 \\
\hline Oleic acid (C18:1 n-9 Z) & 250-1000 & $18.953 \pm 0.006$ & $y=425.2101 x-7612.0155$ & 0.9997 & 0.9993 & 0.2137 & 0.7122 \\
\hline Linolelaidic acid (C18:2 n-6 E) & $250-1000$ & $19.553 \pm 0.006$ & $y=230.1367 x-15194.7850$ & 0.9997 & 0.9994 & 0.2214 & 0.7380 \\
\hline Linoleic acid (C18:2 n-6 Z) & $250-1000$ & $19.920 \pm 0.000$ & $y=223.0317 x-11160.5665$ & 1.0000 & 0.9999 & 0.6918 & 2.3060 \\
\hline$\gamma$-linolenic acid (C18:3 n-6, GLA) & $250-1000$ & $20.580 \pm 0.000$ & $y=212.2357 x-12443.1135$ & 1.0000 & 0.9999 & 0.7184 & 2.3946 \\
\hline$\alpha$-linolenic acid (C18:3 n-3, ALA) & $250-1000$ & $21.160 \pm 0.000$ & $y=202.2944 x-9492.2895$ & 1.0000 & 1.0000 & 1.8403 & 6.1344 \\
\hline Arachidic acid (C20:0) & $250-1000$ & $21.973 \pm 0.015$ & $y=494.0689 x-16256.6330$ & 0.9991 & 0.9983 & 0.1345 & 0.4482 \\
\hline Cis-11-eicosenoic acid (C20:1) & 250-1000 & $22.263 \pm 0.006$ & $y=241.1562 x-12764.6795$ & 1.0000 & 1.0000 & 0.8160 & 2.7199 \\
\hline Cis-11,14-eicosadienoic acid (C20:2) & $250-1000$ & $23.130 \pm 0.010$ & $y=211.9830 x-9814.0900$ & 0.9998 & 0.9996 & 0.2733 & 0.9110 \\
\hline Heneicosanoic acid (C21:0) & $250-1000$ & $23.590 \pm 0.010$ & $y=150.6156 x-2443.3555$ & 1.0000 & 1.0000 & 1.6576 & 5.5254 \\
\hline Cis-8,11,14-eicosatrienoic acid (C20:3 n-6, DGLA) & $250-1000$ & $23.687 \pm 0.006$ & $y=197.8801 x-7653.1285$ & 0.9998 & 0.9996 & 0.2765 & 0.9218 \\
\hline Arachidonic acid (C20:4 n-6, AA) & 250-1000 & $24.103 \pm 0.006$ & $y=194.4791 x-7437.3125$ & 0.9998 & 0.9995 & 0.2568 & 0.8560 \\
\hline Cis-11,14,17-eicosatrienoic acid (C20:3) & 250-1000 & $24.253 \pm 0.006$ & $y=141.3401 x-3637.9235$ & 0.9992 & 0.9984 & 0.1398 & 0.4659 \\
\hline Behenic acid (C22:0) & 250-1000 & $25.180 \pm 0.010$ & $y=408.6182 x-10400.1250$ & 1.0000 & 0.9999 & 0.7288 & 2.4292 \\
\hline Erucic acid $(\mathrm{C} 22: 1 \mathrm{n}-9)$ & $250-1000$ & $25.313 \pm 0.006$ & $y=221.7645 x-17072.2935$ & 1.0000 & 0.9999 & 0.7788 & 2.5959 \\
\hline 5,8,11,14,17-eicosapentadienoic acid (C20:5 n-3, EPA) & $250-1000$ & $25.407 \pm 0.006$ & $y=199.8515 x-10168.5900$ & 0.9997 & 0.9994 & 0.2339 & 0.7797 \\
\hline Cis-13,16-docosadienoic acid (C22:2) & $250-1000$ & $26.173 \pm 0.006$ & $y=213.4314 x-12761.5080$ & 1.0000 & 0.9999 & 0.6514 & 2.1713 \\
\hline Tricosanoic acid $(\mathrm{C} 23: 0)$ & 250-1000 & $26.710 \pm 0.010$ & $y=228.7863 x-7187.5190$ & 0.9997 & 0.9994 & 0.2318 & 0.7728 \\
\hline Lignoceric acid (C24:0) & $250-1000$ & $28.213 \pm 0.006$ & $y=443.1121 x-25091.8985$ & 0.9999 & 0.9999 & 0.5220 & 1.7401 \\
\hline Nervonic acid (C24:1) & $250-1000$ & $28.373 \pm 0.006$ & $y=228.3534 x-11611.2500$ & 1.0000 & 1.0000 & 1.0994 & 3.6648 \\
\hline Cis-4,7,10,13,16,19-docosahexadienoic acid (C22:6 n-3, DHA) & $250-1000$ & $28.630 \pm 0.000$ & $y=215.8342 x-19113.3950$ & 1.0000 & 0.9999 & 0.5976 & 1.9921 \\
\hline
\end{tabular}

$\mathrm{t}_{\mathrm{r}}$ : retention time; SD: standard deviation; $R$ : correlation coefficient; $R^{2}$ : squared correlation coefficient; LOD: limit of detection; LOQ: limit of quantification. 


\subsection{Fatty Acid Profile}

The fatty acid composition of colostrum, transitional, and mature milk samples of women from Granada, as well as that of infant formulas marketed are presented in Tables 3-5. Of the thirty-five standard fatty acids, a total of twenty-eight fatty acids were identified and quantified in our human milk and infant formula samples using GC-MS/MS analysis. These were grouped into saturated (SFAs), monounsaturated (MUFAs), and polyunsaturated (PUFAs) fatty acids. Figure 2 shows the distribution of the main fatty acid groups in the different samples of human milk and infant formulas.

Table 3. Saturated fatty acid (SFAs) composition of colostrum, transitional, and mature milks, and infant formulas $(\% \mathrm{wt}=w \mathrm{t})$.

\begin{tabular}{ccccc}
\hline Fatty Acids (FAs) & Colostrum & Transitional Milk & Mature Milk & Infant Formula \\
\hline Caprylic acid (C8:0) & $0.72 \pm 0.634$ & $0.51 \pm 0.130$ & $0.64 \pm 0.197$ & $0.77 \pm 0.271$ \\
Capric acid (C10:0) & $0.18^{\mathrm{a}, \mathrm{b}, \mathrm{d}} \pm 0.160$ & $1.06 \pm 0.268$ & $1.06 \pm 0.173$ & $1.04 \pm 0.424$ \\
Lauric acid (C12:0) & $1.43^{\mathrm{a}, \mathrm{b}, \mathrm{d} \pm 0.768}$ & $4.65^{\mathrm{e}} \pm 1.578$ & $3.82^{\mathrm{f}} \pm 1.158$ & $7.45 \pm 2.378$ \\
Tridecanoic acid (C13:0) & 0.00 & $0.01 \pm 0.020$ & 0.00 & $0.01 \pm 0.010$ \\
Myristic acid (C14:0) & $3.79 \pm 1.165$ & $4.56 \pm 1.420$ & $3.68 \pm 1.326$ & $3.43 \pm 1.517$ \\
Pentadecanoic acid (C15:0) & $0.15 \pm 0.140$ & $0.22 \pm 0.009$ & $0.18 \pm 0.093$ & $0.15 \pm 0.282$ \\
Palmitic acid (C16:0) & $23.75^{\mathrm{a}, \mathrm{b}} \pm 2.032$ & $20.76^{\mathrm{c}} \pm 2.031$ & $18.13^{\mathrm{f}} \pm 2.332$ & $23.09 \pm 2.669$ \\
Margaric acid (C17:0) & $0.09 \pm 0.133$ & $0.07 \pm 0.104$ & $0.08^{\mathrm{f}} \pm 0.109$ & $0.05 \pm 0.109$ \\
Stearic acid (C18:0) & $6.23^{\mathrm{d}} \pm 1.192$ & $5.54^{\mathrm{e}} \pm 0.906$ & $5.46^{\mathrm{f}} \pm 0.781$ & $3.81 \pm 0.516$ \\
Arachidic acid (C20:0) & $0.04^{\mathrm{d}} \pm 0.096$ & $0.02^{\mathrm{e}} \pm 0.074$ & $0.03^{\mathrm{f}} \pm 0.075$ & $0.33 \pm 0.110$ \\
Lignoceric acid (C24:0) & $0.04^{\mathrm{d}} \pm 0.144$ & 0.00 & 0.00 & 0.00 \\
\hline SFA & $36.05 \pm 5.266$ & $37.49 \pm 3.153$ & $33.19 \pm 4.899$ & $37.31 \pm 4.522$ \\
SC-SFA (C8-C10) & $0.97^{\mathrm{a}, \mathrm{b}, \mathrm{d}} \pm 0.622$ & $1.51 \pm 0.346$ & $1.70 \pm 0.266$ & $2.01 \pm 0.465$ \\
MC-SFA (C12-C16) & $27.71^{\mathrm{d}} \pm 3.305$ & $30.38^{\mathrm{c}} \pm 2.856$ & $26.05^{\mathrm{f}} \pm 4.370$ & $33.76 \pm 4.219$ \\
LC-SFA (> C17) & $6.41^{\mathrm{b}, \mathrm{d}} \pm 1.153$ & $5.35 \pm 0.901$ & $5.55^{\mathrm{f}} \pm 0.735$ & $4.15 \pm 0.451$ \\
\hline
\end{tabular}

SD: standard deviation; SFA: saturated FAs; SC-SFA: short-chain SFA; MC-SFA: medium-chain SFA; LC-SFA: long-chain SFA. (a) Significant differences $(p<0.05)$ between colostrum and transitional milk groups, (b) significant differences $(p<0.05)$ between colostrum and mature milk groups, (c) significant differences $(p<0.05)$ between transitional and mature milk groups, $(\mathrm{d})$ significant differences $(p<0.05)$ between colostrum and infant formula groups, (e) significant differences $(p<0.05)$ between transitional and infant formula groups, (f) significant differences $(p<0.05)$ between mature and infant formula groups.

Table 4. Monounsaturated fatty acid (MUFAs) composition of colostrum, transitional, mature milk, and infant formulas (\% wt $=w t)$.

\begin{tabular}{ccccc}
\hline Fatty Acids (FAs) & Colostrum & Transitional Milk & Mature Milk & Infant Formula \\
\hline Myristoleic acid (C14:1) & $0.02^{\mathrm{a}} \pm 0.064$ & $0.08 \pm 0.085$ & $0.06 \pm 0.0796$ & $0.18 \pm 0.328$ \\
Cis-10-pentadecenoic acid (C15:1) & 0.00 & 0.00 & 0.00 & $0.01 \pm 0.034$ \\
Palmitoleic acid (C16:1 n-9 Z) & $1.52^{\mathrm{d}} \pm 0.320$ & $1.23^{\mathrm{e}} \pm 0.667$ & $1.45^{\mathrm{f}} \pm 0.416$ & $0.37 \pm 0.159$ \\
Vaccenic acid (C18:1 n-7 E) & $1.42^{\mathrm{a}, \mathrm{b}, \mathrm{d}} \pm 0.148$ & $1.12^{\mathrm{e}} \pm 0.161$ & $1.11^{\mathrm{f}} \pm 0.099$ & $0.47 \pm 0.103$ \\
Oleic acid (C18:1 n-9 Z) & $42.11 \pm 5.228$ & $39.81 \pm 4.608$ & $43.88 \pm 7.041$ & $43.54 \pm 3.428$ \\
Gadoleic acid (C20:1 n-9) & $0.97^{\mathrm{a}, \mathrm{b}, \mathrm{d}} \pm 0.253$ & $0.52^{\mathrm{e}} \pm 0.173$ & $0.38 \pm 0.097$ & $0.36 \pm 0.046$ \\
Erucic acid (C22:1 n-9) & 0.00 & $0.03 \pm 0.066$ & $0.01 \pm 0.035$ & 0.00 \\
\hline MUFA & $45.99 \pm 5.750$ & $43.34 \pm 4.754$ & $46.99 \pm 6.796$ & $44.86 \pm 3.689$ \\
\hline
\end{tabular}

SD: standard deviation; MUFA: monounsaturated fatty acids. (a) Significant differences $(p<0.05)$ between colostrum and transitional milk groups, (b) significant differences $(p<0.05)$ between colostrum and mature milk groups, (c) significant differences $(p<0.05)$ between transitional and mature milk groups, (d) significant differences $(p<0.05)$ between colostrum and infant formula groups, (e) significant differences $(p<0.05)$ between transitional and infant formula groups, (f) significant differences $(p<0.05)$ between mature and infant formula groups. 
Table 5. Polyunsaturated fatty acid (PUFAs) composition of colostrum, transitional, and mature milk, and infant formulas $(\% \mathrm{wt}=w \mathrm{t})$.

\begin{tabular}{|c|c|c|c|c|}
\hline Fatty Acids (FAs) & Colostrum & Transitional Milk & Mature Milk & Infant Formula \\
\hline Cis-9,12-hexadecadienoic (C16:2 n-4) & 0.00 & 0.00 & $0.01 \pm 0.032$ & 0.00 \\
\hline Linolelaidic acid (C18:2 n-6 E) & $0.01 \pm 0.047$ & 0.00 & 0.00 & $0.03 \pm 0.072$ \\
\hline Linoleic acid (C18:2 n-6 Z (LA)) & $12.32 \pm 2.643$ & $16.10 \pm 5.325$ & $15.38 \pm 5.754$ & $15.31 \pm 2.667$ \\
\hline$\gamma$-linolenic acid (C18:3 n-6 (GLA)) & $0.00^{\mathrm{a}, \mathrm{b}}$ & $0.04^{\mathrm{e}} \pm 0.064$ & $0.07^{\mathrm{f}} \pm 0.087$ & 0.00 \\
\hline$\alpha$-linolenic acid (C18:3 n-3 (ALA)) & $0.33^{\mathrm{d}} \pm 0.103$ & $0.48^{\mathrm{e}} \pm 0.149$ & $0.45^{\mathrm{f}} \pm 0.121$ & $1.64 \pm 0.247$ \\
\hline Cis 11,14-eicosadienoic acid (C20:2) & $0.98^{\mathrm{a}, \mathrm{b}, \mathrm{d}} \pm 0.282$ & $0.64^{\mathrm{e}} \pm 0.332$ & $0.40^{\mathrm{f}} \pm 0.084$ & 0.00 \\
\hline Dihono- $\gamma$-linolenic acid (C20:3 n-6 (DGLA)) & $0.74^{\mathrm{a}, \mathrm{b}, \mathrm{d}} \pm 0.274$ & $0.49^{\mathrm{e}} \pm 0.139$ & $0.34^{\mathrm{f}} \pm 0.098$ & 0.00 \\
\hline Arachidonic acid (C20:4 n-6 (AA)) & $0.72^{\mathrm{a}, \mathrm{b}, \mathrm{d}} \pm 0.321$ & $0.46 \pm 0.114$ & $0.36 \pm 0.073$ & $0.31 \pm 0.078$ \\
\hline Cis 13,16 -docosadienoic acid (C22:2) & $0.03 \pm 0.084$ & 0.00 & 0.00 & 0.00 \\
\hline Cis $4,7,10,13,16,19$-docosahexadienoic acid (C22:6 n-3 (DHA)) & $0.47 \pm 0.240$ & $0.36 \pm 0.140$ & $0.33 \pm 0.240$ & $0.22 \pm 0.099$ \\
\hline PUFA & $15.94 \pm 2.504$ & $17.80 \pm 5.038$ & $17.48 \pm 5.828$ & $17.55 \pm 2.667$ \\
\hline UFA & $62.68 \pm 6.83$ & $63.07 \pm 3.62$ & $65.78 \pm 5.88$ & $60.94 \pm 6.22$ \\
\hline SFA/UFA & $0.62 \pm 0,20$ & $0.59 \pm 0,09$ & $0.53 \pm 0,14$ & $0.64 \pm 0.17$ \\
\hline $\mathrm{n}-3$ PUFA & $0.77^{\mathrm{d}} \pm 0.308$ & $0.84^{\mathrm{e}} \pm 0.165$ & $0.79^{\mathrm{f}} \pm 0.350$ & $1.81 \pm 0.232$ \\
\hline n-6 PUFA & $14.11^{\mathrm{a}} \pm 2.333$ & $16.38 \pm 4.979$ & $16.14 \pm 5.907$ & $14.83 \pm 1.756$ \\
\hline LC-PUFA (C20-C24) & $3.06^{\mathrm{a}, \mathrm{b}, \mathrm{d}} \pm 0.641$ & $1.85^{\mathrm{c}, \mathrm{e}} \pm 0.381$ & $1.43^{\mathrm{f}} \pm 0.260$ & $0.54 \pm 0.158$ \\
\hline LA/ALA & $38.15^{\mathrm{d}} \pm 17.980$ & $32.03^{\mathrm{e}} \pm 10.030$ & $31.30^{\mathrm{f}} \pm 15.370$ & $9.53 \pm 0.667$ \\
\hline AA/DHA & $1.74 \pm 0.812$ & $1.54 \pm 0.683$ & $1.43 \pm 1.016$ & $1.33 \pm 0.361$ \\
\hline
\end{tabular}

SD: standard deviation; PUFA: polyunsaturated fatty acids; UFA: unsaturated FAs; SFA/UFA: saturated FAs / unsaturated FAs; LC-PUFA: long-chain PUFA; LA/ALA: linoleic acid/ $\alpha$-linolenic acid; AA/DHA: araquidonic acid/docoxahexaenoic acid. (a) Significant differences $(p<0.05)$ between colostrum and transitional milk groups, (b) significant differences $(p<0.05)$ between colostrum and mature milk groups, (c) significant differences $(p<0.05)$ between transitional and mature milk groups, (d) significant differences $(p<0.05)$ between colostrum and infant formula groups, (e) significant differences $(p<0.05)$ between transitional and infant formula groups, (f) Significant differences $(p<0.05)$ between mature and infant formula groups.

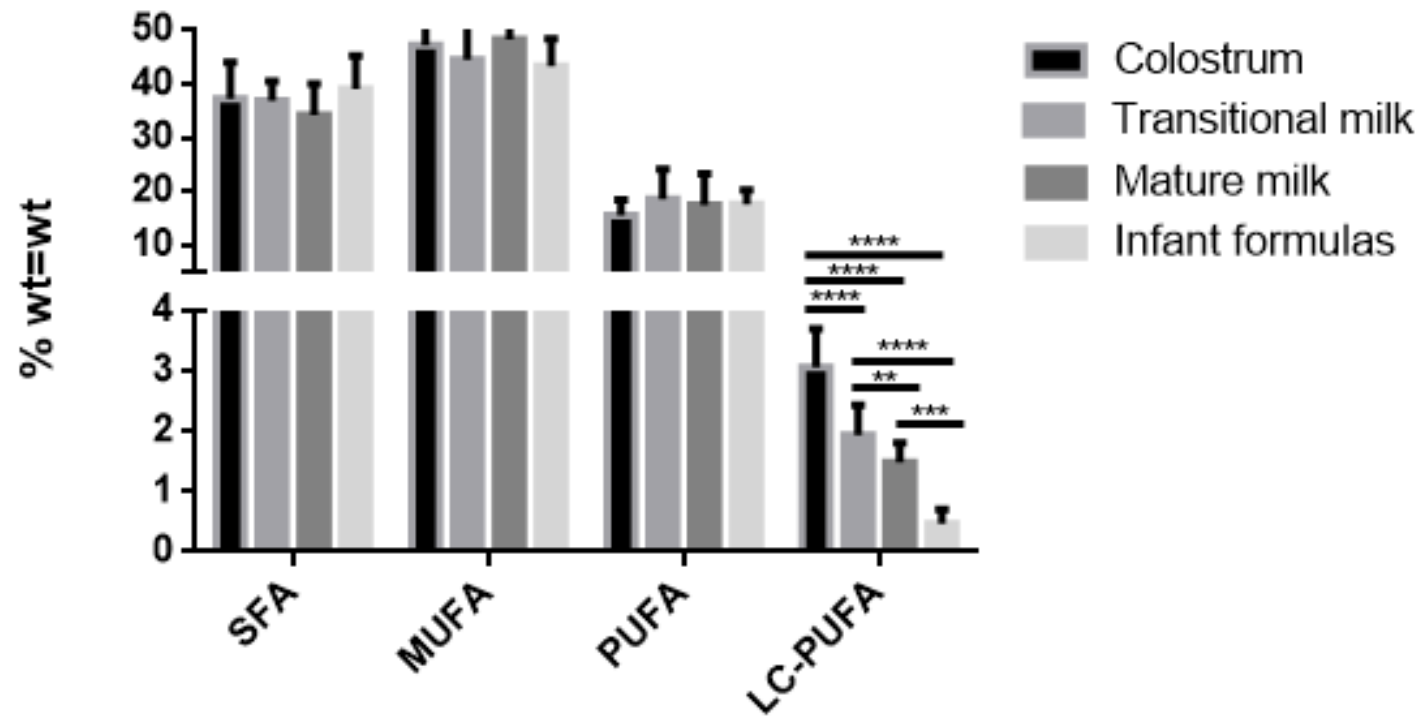

Fatty Acids

Figure 2. Fatty acid profile in human milk (colostrum $n=13$; transitional milk $n=13$; and mature milk $\mathrm{n}=12$ ) and infant formulas ( $\mathrm{n}=7$ ). SFA: saturated fatty acids; MUFA: monounsaturated fatty acids; PUFA: polyunsaturated fatty acids and LC-PUFA: long-chain polyunsaturated fatty acids. Significant differences $\left({ }^{* *} p<0.01\right)$, $\left.{ }^{* * *} p<0.001\right)$ and $\left.{ }^{* * * *} p<0.0001\right)$.

\subsubsection{Saturated Fatty Acids (SFAs)}

The saturated fatty acids identified are shown in Table 3.

The major SFA was C16:0, which is seen to decrease significantly when comparing the colostrum group to the transitional group $(p<0.05)$ and the mature group $(p<0.0001)$ within the milk samples (23.75\%, $20.76 \%$ to $18.13 \%$ respectively). Nevertheless, a significant increase was observed between the colostrum group and the transitional and mature milk group $(p<0.0001)$ for $\mathrm{C} 10: 0(0.18 \% ; 1.06 \%$, and $1.06 \%$, respectively) and C12:0 (1.43\%; $4.65 \%$, and $3.82 \%$, respectively). 
Infant formulas showed significantly lower values of C18:0 than the colostrum $(p<0.0001)$, transitional $(p<0.01)$, and mature $(p<0.01)$ groups. In contrast, higher values of C12: 0 were found in infant formulas than the colostrum $(p<0.0001)$, transitional $(p<0.01)$, and mature $(p<0.001)$ groups. In addition, higher levels of C10: 0 were found relative to colostrum group $(p<0.0001)$, and higher C16: 0 relative to the transitional $(p<0.05)$ and mature $(p<0.001)$ groups.

Thus, infant formulas have a higher proportion of SC-SFA $(0.97 \%$ for colostrum, $1.51 \%$ for transitional milk, $1.70 \%$ for mature milk, and $2.01 \%$ for infant formulas) and MC-SFA $(27.71 \%$ for colostrum, $30.38 \%$ for transitional milk; $26.05 \%$ for mature milk and $33.76 \%$ for infant formulas) than human milk. In contrast, they have a lower percentage of LC-SFA $(6.41 \%$ for colostrum, $5.35 \%$ for transitional milk, $5.55 \%$ for mature milk, and $4.15 \%$ for infant formulas).

The other saturated fatty acids and overall SFA did not show significant differences between the milk groups.

\subsubsection{Monounsaturated Fatty Acids (MUFAs)}

The monounsaturated fatty acids identified are shown in Table 4.

The major MUFA was C18:1 n-9 Z, with similar values being found between human milk groups and infant formulas.

There was a decreasing trend between the colostrum group to the transitional and mature milk groups for C16:1 n-9 Z (1.52\%, 1.23\%, and 1.45\%, respectively); C18:1 n-7 E $(1.42 \%, 1.12 \%$, and $1.11 \%$, respectively); and C20:1 n-9 $(0.97 \%, 0.52 \%$, and $0.38 \%$, respectively).

In addition, significantly higher percentages were observed for these three fatty acids in human milk samples than in infant formulas $(p<0.01)(0.37 \%$ for $\mathrm{C} 16: 1 \mathrm{n}-9 \mathrm{Z} ; 0.47 \%$ for $\mathrm{C} 18: 1 \mathrm{n}-7 \mathrm{E}$; and $0.36 \%$ for C20:1 n-9).

The other monounsaturated fatty acids and the total MUFA did not show significant differences between the milk groups and infant formulas.

\subsubsection{Polyunsaturated Fatty Acids (PUFAs)}

The polyunsaturated fatty acids identified are shown in Table 5.

The major PUFA was C18:2 n-6 Z, (LA), with similar values being found between human milk groups and infant formulas. In contrast, $\mathrm{C} 18: 3 \mathrm{n}-3$ (ALA) showed significantly higher values in infant formula than in the different human milk groups $(p<0.0001)$. Thus, the LA/ALA ratio showed statistically significant differences between the human milk group and infant formulas $(p<0.001)$.

C20:2 and C20:3 n- 6 showed a significant decrease between the colostrum group and the transitional $(p<0.01)$ and mature $(p<0.0001)$ milk groups. Whilst measurements for C22:6 n-3 (DHA) did not show statistically significant differences, instead presenting similar trends between groups.

With regards to LC-PUFA (C20-24), greater variability was seen between the human milk and infant formula groups. Figure 3 shows the variability of the main PUFAs in human milk and infant formulas. The latter group did not contain C20:2, GLA, and DGLA, whilst the percentages of AA and DHA were significantly lower than that found in human milk.

The remaining polyunsaturated fatty acids and overall PUFA did not show significant differences between the milk groups and infant formulas. 


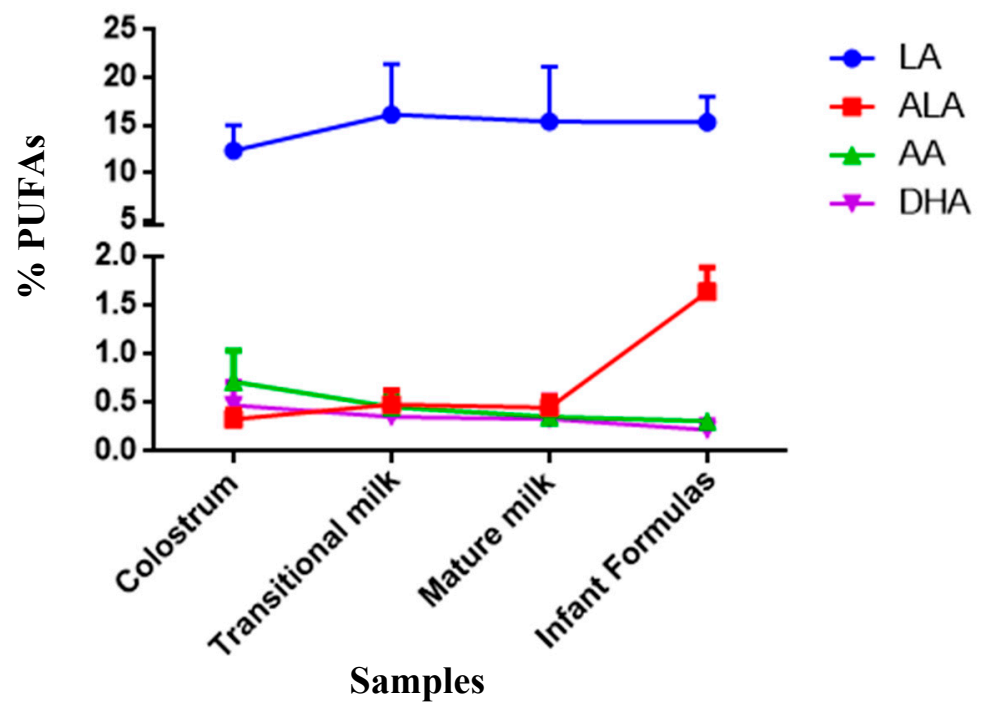

Figure 3. Trend of the majority of polyunsaturated fatty acids (PUFAs) in human milk (colostrum, transitional, and mature milk) and infant formulas. LA: linoleic acid; ALA: $\alpha$-linolenic acid; AA: araquidonic acid; DHA: docoxahexaenoic acid.

\subsection{Discriminant Analysis}

Forty-five cases were used to develop a model that discriminates between the human milk and infant formula samples. Thirty-six predictor variables were introduced. Table 6 displays the discriminant functions obtained in the present analysis.

Table 6. Discriminant functions that predict the types of milks analyzed based on fatty acid levels.

\begin{tabular}{ccccccc}
\hline $\begin{array}{c}\text { Discriminant } \\
\text { Functions }\end{array}$ & Eigenvalue & $\begin{array}{c}\text { Relative } \\
\text { Percentage (\%) }\end{array}$ & $\begin{array}{c}\text { Canonical } \\
\text { Correlation }\end{array}$ & $\begin{array}{c}\text { Lambda De } \\
\text { Wilks }\end{array}$ & Chi-Squared & $p$-Value \\
\hline 1 & 126.3990 & 87.58 & 0.9961 & 0.0002 & 210.5987 & $0.0000^{*}$ \\
2 & 15.9167 & 11.03 & 0.9610 & 0.0197 & 94.2629 & $0.0282^{*}$ \\
3 & 2.0021 & 1.39 & 0.8166 & 0.3331 & 26.3837 & 0.8214 \\
\hline
\end{tabular}

*: Significant differences $(p<0.05)$.

In this case, our first discriminant function has a relative variance percentage close to $100 \%$ $(87.58 \%)$, while the second and third discriminant function is only able to explain $11.03 \%$ and $1.39 \%$ of the variance of the data, respectively. The $p$-value of discriminant functions 1 and 2 are less than 0.05 , whereby both discriminant functions are statistically significant with a 95.0\% confidence level.

In addition, the canonical correlation of the first and second functions is closer to 1 . These functions allowed $91.1 \%$ of groups to be classified (100\% for infant formulas versus $83.3 \%$ of mature human milk). Finally, Wilk's Lambda is also closer to 0 in the first two discriminant functions.

Taken together, results indicate that our first two discriminant functions are capable of separating the data much better than the third function.

A graphical representation of the two dimensions is shown in Figure 4, according to the first and second discriminating functions. 


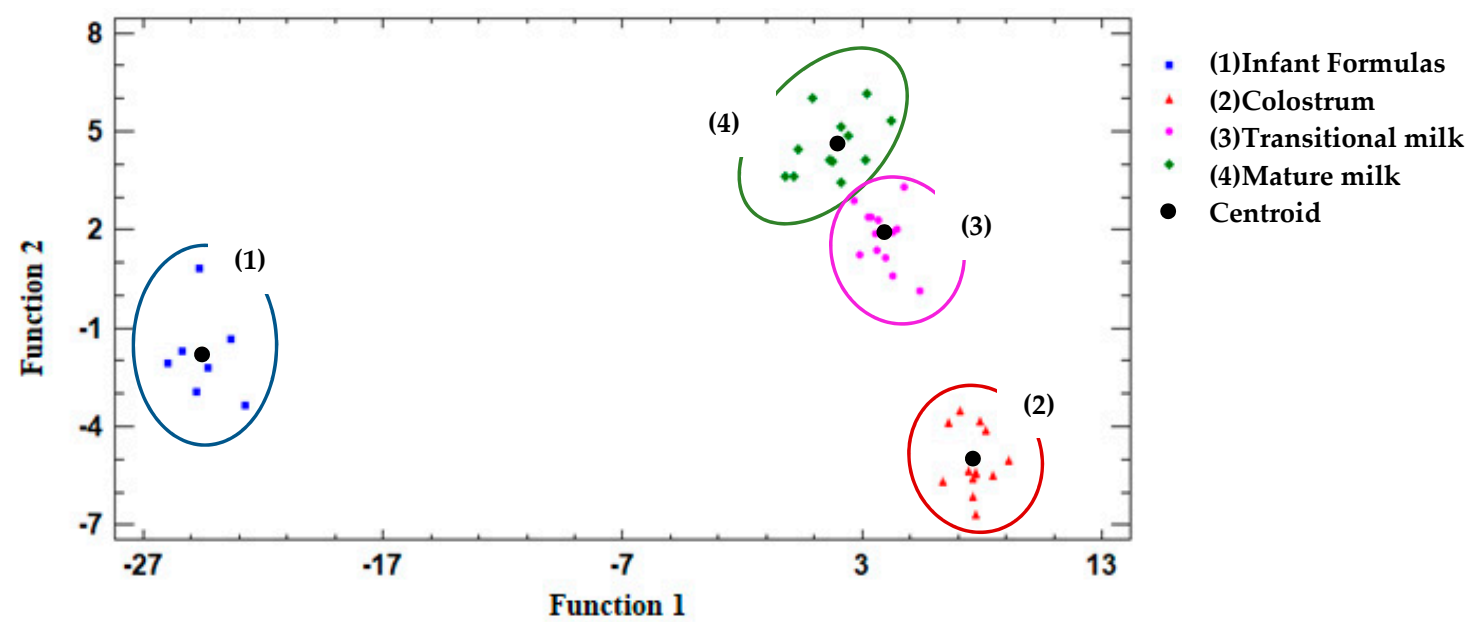

Figure 4. Representation of the different groups of milks studied (colostrum, transitional milk, mature milk, and infant formulas) based on the two significant functions according to the discriminant analysis performed. Centroid: average value of the discriminant function for each one of the samples.

\section{Discussion}

The results of the present study show that the average characteristics relating to the fatty acid composition of human milk can be summarized as follows. Palmitic acid $(\approx 20 \%)$, oleic acid $(\approx 44 \%)$, and linoleic acid $(\approx 15 \%)$ were the predominant fatty acids in the colostrum, transitional, and mature milk groups, and in the infant formulas. These represented between $76.67 \%$ and $81.94 \%$ of the total fatty acids. This trend is similar to that found by Yang et al., which was between $77.98 \%$ and $78.57 \%$ [22]. Next, we consider the results relating to myristic acid $(\approx 4 \%)$ and stearic acid $(\approx 6 \%)$.

All of these values were very similar to those described by other authors. Yang et al. [23] found similar values in palmitic acid (21.77\%), myristic acid (3.91\%), and stearic acid (5.14\%); but found lower values in oleic acid (33.32\%) and higher values in linoleic acid (22.54\%). However, Zou et al. [24] reported more similar values with respect to palmitic acid $(20.3 \%)$, linoleic acid $(17.1 \%)$, myristic acid $(4.9 \%)$, and stearic acid (6.0\%). On the other hand, they also identified lower percentages of oleic acid (34.9\%).

\subsection{Saturated Fatty Acids (SFAs)}

Total saturated fatty acid (SFA) content remained stable across all of the milk groups [24]. Human milk typically contains approximately $34 \%$ to $47 \%$ saturated fatty acids, mainly palmitic acid $(17-25 \%)$ [7,22]. Similar values were found in other studies $[10,13,25]$. The colostrum and infant formula groups presented similar percentages of this SFA ( $23.75 \%$ and $23.09 \%$, respectively). However, in human milk, palmitic acid is esterified with triglycerides in position 2 (position $\beta$ ), whereas unmodified milk fat of infant formulas is esterified in positions 1 and 3 . The specific distribution of fatty acids in the triglyceride plays a key role in the digestion and absorption of lipids. It appears that this modification of fat decreases the stability and quantity of calcareous soaps in feces, thus decreasing its consistency [24-26].

Other fatty acids, such as C10:0 and C12:0 increased from one lactation stage to the next. This trend was very similar to that found in another Spanish study [10] $(0.66 \%, 1.66 \%$, and $1.63 \%)$ for C10:0 and $(3.49 \%, 6.97 \%$, and $6.28 \%)$ for C12:0 in colostrum, transitional, and mature milk, respectively.

Therefore, infant formulas have a higher proportion of SC-SFA and MC-SFA than human milk, possibly due to the addition of vegetable oils [26]. MC-SFA are commonly supplemented and incorporated into these infant formulas because they can be directly absorbed by the portal vein and rapidly generate energy for infants [27]. However, this has been related to an increase in the level of total and low-density lipoprotein (LDL) cholesterol concentration in plasma leading to a high risk of cardiovascular disease. On the other hand, LC-SFA is reported to be neutral concerning its effects on lipoprotein cholesterol levels [28]. 
Despite this, overall lauric and myristic acid content is recommended to not exceed $20 \%$ of total fat in infant formulas, with content being $10.88 \%$ in the present case [29].

\subsection{Monounsaturated Fatty Acids (MUFAs)}

Total monounsaturated fatty acid (MUFA) remained stable in all milk samples $(45.99 \%$ for colostrum, $43.34 \%$ for transitional, and $46.99 \%$ for mature milk group). This trend has also been described by other authors [30]. Barreito et al. reported a percentage of $44.1 \%$.

Oleic acid (C18:1 n-9) constitutes more than 90\% of the total MUFAs, finding similar values in human milk groups and infant formulas. This is fundamentally linked to the consumption of olive oil, representing levels greater than $40 \%$ [6,15].

These values are higher than those found in other studies, both Spanish and European studies, possibly because southern Spain shows higher levels of adherence to the Mediterranean diet [31]. This may be due to the potentially high adherence to the Mediterranean diet of our participants which is composed of foods rich in oleic acid, especially for the high consumption of extra virgin oil. According to the data offered by ministry of agriculture, food and environment (MAGRAMA) in 2018, Andalusía purchases and consumes more extra virgin olive oil than any other autonomous community, accounting for $25.99 \%$ of the volume distributed across Spain (L) [32].

C16:1 n-9, C18:1 n-7, and C20:1 n-9 were the three most abundant MUFAs in human milk after oleic acid, with a percentage of around 3-4\%, whilst the percentage present in infant formulas was $1.2 \%$.

The results of a previous study indicate that the majority of commercially available IF do not contain scientifically recommended amounts of vaccenic acid, and that their fatty acid composition is deficient in comparison with human milk [33]. Vaccenic acid is the major trans-fatty acid in ruminant milk fat. It is unique in that it may provide cis-9, trans-11-octodecadienoic (cis 9, trans 11-C18:2; also known as rumenic acid) to the consumer through endogenous desaturation by the $\Delta-9$ desaturase enzyme [34]. Rumenic acid is the most common conjugated linoleic acid (CLA) isomer and it has been shown to promote various beneficial health-related effects, including anti-carcinogenic, anti-atherosgenic, anti-diabetic, and immune-modulating effects, in addition to effects on body composition and fat metabolism [35-37].

\subsection{Polyunsaturated Fatty Acids (PUFAs)}

Concentrations of PUFA in human milk are relatively stable during the first year of life. AA typically constitutes $1 \%(0.72 \%)$ in colostrum and $0.5 \%$ in mature milk $(0.36 \%)$; DHA is approximately equivalent to $0.5 \%$ in colostrum $(0.47 \%)$ and $0.25 \%$ in mature milk (0.33\%) [16]. Also, it typically contains approximately $12 \%$ to $26 \%$ n-6 PUFA and $0.8 \%$ to $3.6 \%$ n-3 PUFA [7].

Moreover, essential fatty acid (EFA) content varies depending on the stage of lactation, particularly LA levels (12.32\% for colostrum; $16.10 \%$ for transitional; and $15.38 \%$ for mature milk). Zou et al. showed this trend in the results of their study $(21.01 \%, 21.05 \%$, and $25.58 \%$ for colostrum, transitional, and mature milk, respectively) [23], although the values of our study were lower. Values, however, were similar to those reported by Ribeiro et al. [38] (15.46\% for samples 7 days postpartum and $16.20 \%$ for samples at 4 weeks postpartum).

DHA, AA, and DGLA content also showed a decrease according to the stage of lactation [38,39], finding higher values in colostrum than in transitional and mature milk groups [23]. Only a slight increase in GLA was observed, correlated with a reduction in inflammation after childbirth. GLA is the substrate for DGLA synthesis, another anti-inflammatory fatty acid $[36,37,40]$ Fu et al. evaluated the DHA and arachidonic acid (AA) levels in human milk according to country and region, reporting similar values $(0.42 \%$ for DHA and $0.71 \%$ for $\mathrm{AA})$ for Spain to those found in the present study [41].

However, higher levels of ALA were observed in mature milk compared to the colostrum and transitional groups, as has also been described by other authors [15].

In infant formulas, LA percentage $(15.31 \%)$ was similar to that found in human milk $(14.6 \%)$. However, the content of AA $(0.31 \%)$ and DHA $(0.22 \%)$ was lower than in human milk $(0.51 \%$ and $0.39 \%$, 
respectively), especially when considering the colostrum stage. Conversely, ALA values were also much higher in infant formulas than in human milk ( $1.64 \%$ and $0.42 \%$, respectively). DHA experiences a longer and more complicated synthesis which limits the conversion rate of ALA/DHA [42]. This trend is shown in Figure 2.

For this reason, infant formulas present a significantly higher percentage of n-3 PUFA than that seen in the different groups of human milk. This is due to their high amount of $\alpha$-linolenic acid.

Codex Alimentarius stipulate that the AA and DHA content of infant formulas should, at least, have the same concentration [43]. The content of these fatty acids in our infant formulas was $0.31 \%$ and $0.22 \%$, respectively. These values being similar to those described by Chen et al. [27], which reported values of $0.41 \%$ for AA and $0.23 \%$ for DHA.

These values are below estimated averages for AA and DHA in human milk samples studied by Brenna et al. [42]. These authors included 84 studies and reported that the worldwide mean concentration in human milk was $0.47 \% \pm 0.13 \%$ for AA and $0.32 \% \pm 0.22 \%$ for DHA. These values are very similar to those uncovered in the present sample ( $0.51 \%$ for AA and $0.39 \%$ for DHA).

The AA/DHA ratio did not vary with increasing milk maturation, however the LA/ALA ratio was significantly higher in the colostrum, transitional, and mature milk groups than in infant formulas $(38.15 \%, 32.03 \%, 31.30 \%$, and $9.53 \%$, respectively). It is important to note that this ratio is within the guideline range of 5:1 and 15:1 suggested by the ESPHGAN Committee on Nutrition (European Society for Pediatric Gastroenterology Hepatology and Nutrition) [29].

\subsection{Discriminant Analysis}

An eigenvalue in discriminant analysis is the characteristic root of each function, that is, it is an indication of how well that function differentiates the groups, and the larger the eigenvalue is, the better the function differentiates the groups [44].

In the present study, the infant formula group shows left-handside displacement on the graph, while the human milk groups are found to the right of the graph. Differences between the colostrum, transitional, and mature human milk groups can be established.

This indicates that all of the present samples can be grouped and differentiated from each other according to their fatty acid profile. Infant formulas are distinct from the human milk groups, largely due to the differences described in their LC-PUFAs. In addition, it is interesting to observe that the different human milk samples can be assigned to independent groups, following the observation that samples belonging to the colostrum group have a different composition than the other samples (transitional and mature groups).

In conclusion, this method was proven to be a useful tool for studying the relationships between oils according to their fat composition [44].

\subsection{Limitations and Strenghts}

Some limitations should be acknowledged. The small sample size used in the present study is a limitation, although we did not observe significant differences in the fatty acid profile of lactating women according to the anthropometric variables studied, such as weight, height, or BMI (normal BMI and those with a BMI above $25 \mathrm{~kg} / \mathrm{m}^{2}$ ); this also being the case for the other variables described.

On the other hand, data on human milk has been previously reported for many European countries and cultures but no recent data about milk from lactating women relates to southern Spain $[10,22]$. This means there is an interest in carrying out this study with lactating women in Granada. It also constitutes a strength of the present study that it provides new fatty acid data in human milk, as well as infant formulas that are currently commercial, through the use of novel analytical techniques (GC-MS/MS). 


\section{Conclusions}

The outcome of the present study showed that the fatty acid profile of human milk samples varies throughout lactation. This variability in the profile of fatty acids between the different samples of human milk justifies that is not a static fluid and changes over time, adapting to the nutritional needs of the infant. However, infant formulas are rather uniform with respect to their composition.

According to the results, despite the fatty acid profile being similar in infant formulas and in human milk in terms of total SFA, MUFA, and PUFA; significant differences were found in some important fatty acids (such as ALA, GLA, DHA, or AA) between different human milk groups and infant formulas. Further, more evident statistically significant differences were observed between the colostrum group and the other samples.

Nevertheless, although infant formulas are enriched with the main LC-PUFAs, typically rich in LA, and in some cases with sufficient contributions of ALA, they tend to have low DHA, AA, and GLA content with respect to human milk. These fatty acids are important for fetal growth, and brain and retina development during pregnancy and the early years of life. Scientific evidence has shown that non-breastfed children suffer from a greater prevalence, severity, and longevity of diseases, not only during the time of breastfeeding but many years later.

Furthermore, the distribution of palmitic acid in infant formulas should resemble that of human milk as the specific distribution of fatty acids in triglyceride plays a key role in the digestion and absorption of lipids.

In conclusion, this experimental work can be used to ensure that infant formulas are as similar as possible to human milk and reproduces as close as possible the complexity of human milk composition. This is important in circumstances where breastfeeding is impossible, insufficient, or undesired.

Supplementary Materials: The following are available online at http://www.mdpi.com/2072-6643/11/12/3055/s1, Table S1: Basic formulation details of Infant Formulas (IF).

Author Contributions: The author's responsibilities were as follows: Conceptualization, M.O.-H.; data curation, R.G.-M.; investigation, S.S.-H.; methodology and writing-original draft, S.S.-H. and A.E.-M.; supervision, M.O.-H. and B.M.-B.; writing-review and editing, M.O.-H. and M.J.A.-C.; project administration, M.J.A.-C.

Funding: This project was supported by the Spanish Ministry of Science, Innovation and Universities (Project FIS-ISCIII PI17/02305) and the AGR-279 research group of the Department of Nutrition and Bromatology (University of Granada).

Acknowledgments: The authors thank the volunteers who made the study possible through their participation and collaboration. This paper and results presented constitute part of the thesis of Silvia Sánchez Hernández which is being carried out within the Nutrition and Food Science Doctorate Program at the University of Granada.

Conflicts of Interest: The authors declare that there are no conflicts of interest regarding the publication of this paper.

\section{References}

1. Organización Mundial de la Salud (OMS). Lactancia Materna Exclusiva; WHO: Geneva, Switzerland, 2019.

2. Binns, C.; Lee, M.; Low, W.Y. The Long-Term Public Health Benefits of Breastfeeding. Asia Pac. J. Public Health 2016, 28, 7-14. [CrossRef] [PubMed]

3. Organización Mundial de la Salud (OMS). Organización Panamericana de la Salud (OPS) La Alimentación del Lactante y del Niño Pequeño; OMS: Geneva, Switzerland, 2009; Volume 1, ISBN 9789275330944.

4. Comité de Lactancia Materna de la Asociación Española de Pediatría (AEPED). LACTANCIA MATERNA EN CIFRAS: TASAS DE INICIO Y DURACIÓN DE LA LACTANCIA EN ESPAÑA Y EN OTROS PAÍSES; AEPED: Madrid, Spain, 2016.

5. Lopes, T.I.B.; Cañedo, M.C.; Oliveira, F.M.P.; Alcantara, G.B. Toward Precision Nutrition: Commercial Infant Formulas and Human Milk Compared for Stereospecific Distribution of Fatty Acids Using Metabolomics. OMICS J. Integr. Biol. 2018, 22, 484-492. [CrossRef] [PubMed]

6. Mazzocchi, A.; D’Oria, V.; Cosmi, V.; Bettocchi, S.; Milani, G.; Silano, M.; Agostoni, C.; Mazzocchi, A.; D'Oria, V.; De Cosmi, V.; et al. The Role of Lipids in Human Milk and Infant Formulae. Nutrients 2018, 10, 567. [CrossRef] [PubMed] 
7. Delplanque, B.; Gibson, R.; Koletzko, B.; Lapillonne, A.; Strandvik, B. Lipid Quality in Infant Nutrition. J. Pediatr. Gastroenterol. Nutr. 2015, 61, 8. [CrossRef] [PubMed]

8. Long, A.C.; Kaiser, J.L.; Katz, G.E. Lipids in infant formulas: Current and future innovations. Lipid Technol. 2013, 25, 127-129. [CrossRef]

9. Sioen, I.; van Lieshout, L.; Eilander, A.; Fleith, M.; Lohner, S.; Szommer, A.; Petisca, C.; Eussen, S.; Forsyth, S.; Calder, P.C.; et al. Systematic Review on N-3 and N-6 Polyunsaturated Fatty Acid Intake in European Countries in Light of the Current Recommendations-Focus on Specific Population Groups. Ann. Nutr. Metab. 2017, 70, 39-50. [CrossRef] [PubMed]

10. López-López, A.; López-Sabater, M.; Campoy-Folgoso, C.; Rivero-Urgell, M.; Castellote-Bargalló, A. Fatty acid and sn-2 fatty acid composition in human milk from Granada (Spain) and in infant formulas. Eur. J. Clin. Nutr. 2002, 56, 1242-1254. [CrossRef]

11. Van Elten, T.M.; Van Rossem, L.; Wijga, A.H.; Brunekreef, B.; de Jongste, J.C.; Koppelman, G.H.; Smit, H.A. Breast milk fatty acid composition has a long-term effect on the risk of asthma, eczema, and sensitization. Allergy 2015, 70, 1468-1476. [CrossRef]

12. Hurtado, J.A.; Iznaola, C.; Peña, M.; Ruíz, J.; Peña-Quintana, L.; Kajarabille, N.; Rodriguez-Santana, Y.; Sanjurjo, P.; Aldámiz-Echevarría, L.; Ochoa, J.; et al. Effects of Maternal $\Omega-3$ Supplementation on Fatty Acids and on Visual and Cognitive Development. J. Pediatr. Gastroenterol. Nutr. 2015, 61, 472-480. [CrossRef]

13. Barreiro, R.; Regal, P.; López-Racamonde, O.; Cepeda, A.; Fente, C.A. Comparison of the fatty acid profile of Spanish infant formulas and Galician women breast milk. J. Physiol. Biochem. 2018, 74, 127-138. [CrossRef]

14. Juber, B.A.; Jackson, K.H.; Johnson, K.B.; Harris, W.S.; Baack, M.L. Breast milk DHA levels may increase after informing women: A community-based cohort study from South Dakota USA. Int. Breastfeed. J. 2016, 12, 7. [CrossRef] [PubMed]

15. De Souza Santos da Costa, R.; da Silva Santos, F.; de Barros Mucci, D.; de Souza, T.V.; de Carvalho Sardinha, F.L.; Moutinho de Miranda Chaves, C.R.; das Graças Tavares do Carmo, M. trans Fatty Acids in Colostrum, Mature Milk and Diet of Lactating Adolescents. Lipids 2016, 51, 1363-1373. [CrossRef] [PubMed]

16. Marangoni, F.; Agostoni, C.; Lammard, A.M.; Giovannini, M.; Galli, C.; Riva, E. Polyunsaturated fatty acid concentrations in human hindmilk are stable throughout 12-months of lactation and provide a sustained intake to the infant during exclusive breastfeeding: An Italian study. Br. J. Nutr. 2000, 84, 103-109. [PubMed]

17. EU. Commission Directive 2006/141/EC of 22 December 2006 on Infant Formulae and Follow-on Formulae and Amending Directive 1999/21/EC, 30. 12. 2006; EU: Brussels, Belgium, 2006; pp. 1-33.

18. Lien, E.L.; Richard, C.; Hoffman, D.R. DHA and ARA addition to infant formula: Current status and future research directions. Prostaglandins Leukot. Essent. Fat. Acids 2018, 128, 26-40. [CrossRef] [PubMed]

19. Ministerio de Sanidad, Servicios Sociales e Igualdad. Guía de Práctica Clínica sobre lactancia materna 2017, 321. Available online: https://www.aeped.es/sites/default/files/guia_de_lactancia_materna.pdf (accessed on 19 June 2019).

20. Hara, A.; Radin, N. Lipid Extraction of Tissues. Evaluation 1978, 426, 420-426.

21. Christie, W.W. A simple procedure for rapid transmethylation of glycerolipids and cholesteryl esters. J. Lipid Res. 1982, 23, 1072-1075.

22. He, Y.-B.; Ren, H.-W.; Cao, Y.-T.; Li, H.-J.; Zhang, Z.; Liu, N. Comparing the composition and trend of fatty acid in human milk with bovine milk and infant formula in northeast region of China. CyTA J. Food 2016, 14, 632-638. [CrossRef]

23. Zou, L.; Pande, G.; Akoh, C.C. Infant Formula Fat Analogs and Human Milk Fat: New Focus on Infant Developmental Needs. Annu. Rev. Food Sci. Technol. 2016, 7, 139-165. [CrossRef]

24. Gardner, A.S.; Rahman, I.A.; Lai, C.T.; Hepworth, A.; Trengove, N.; Hartmann, P.E.; Geddes, D.T. Changes in Fatty Acid Composition of Human Milk in Response to Cold-Like Symptoms in the Lactating Mother and Infant. Nutrients 2017, 9, 1034. [CrossRef]

25. Innis, S.M. Palmitic Acid in Early Human Development. Crit. Rev. Food Sci. Nutr. 2016, 56, 1952-1959. [CrossRef]

26. Garcia, C.; Innis, S. Structure of the human milk fat globule. Lipid Technol. 2013, 25, 223-226. [CrossRef]

27. Chen, Y.; Zhang, X.; Li, D.; Yi, H.; Xu, T.; Li, S.; Zhang, L. Fatty acid and triacylglycerol comparison of infant formulas on the Chinese market. Int. Dairy J. 2019, 95, 35-43. [CrossRef] 
28. Di, D.; In, R.; Agrarie, S.E.T. Milk and dairy products: Evaluation of bioactive components. 2014. Available online: https://pdfs.semanticscholar.org/7145/03282aa7dffe66a3c8d0c224daf32d865d4a.pdf (accessed on 19 June 2019).

29. Koletzko, B.; Baker, S.; Cleghorn, G.; Neto, U.F.; Gopalan, S.; Hernell, O.; Hock, Q.S.; Jirapinyo, P.; Lonnerdal, B.; Pencharz, P.; et al. Global Standard for the Composition of Infant Formula: Recommendations of an ESPGHAN Coordinated International Expert Group. J. Pediatric Gastroenterol. Nutr. 2005, 41, 584-599. Available online: https://pdfs.semanticscholar.org/b165/c33c861705c790a4e9620e7d99334e96cd3b.pdf (accessed on 24 July 2019). [CrossRef] [PubMed]

30. Barreiro, R.; Díaz-Bao, M.; Cepeda, A.; Regal, P.; Fente, C.A. Fatty acid composition of breast milk in Galicia (NW Spain): A cross-country comparison. Prostaglandins Leukot. Essent. Fat. Acids 2018, 135, 102-114. [CrossRef]

31. Krešić, G.; Dujmović, M.; Mandić, M.L.; Delaš, I. Relationship between Mediterranean diet and breast milk fatty acid profile: A study in breastfeeding women in Croatia. Dairy Sci. Technol. 2013, 93, 287-301. [CrossRef]

32. Ministerio de Agricultura, P.y.A. Informe del Consumo Alimentario en España 2017. Gob. España $2018,242$.

33. Martysiak-Żurowska, D.; Kiełbratowska, B.; Szlagatys-Sidorkiewicz, A. The content of conjugated linoleic acid and vaccenic acid in the breast milk of women from Gdansk and the surrounding district, as well as in, infant formulas and follow-up formulas. nutritional recommendation for nursing women. Dev. Period Med. 2018, 22, 128-134.

34. Mosley, E.E.; McGuire, M.K.; Williams, J.E.; McGuire, M.A. Cis-9, Trans-11 Conjugated Linoleic Acid Is Synthesized from Vaccenic Acid in Lactating Women. J. Nutr. 2006, 136, 2297-2301. [CrossRef]

35. Turpeinen, A.M.; Mutanen, M.; Aro, A.; Salminen, I.; Basu, S.; Palmquist, D.L.; Griinari, J.M. Bioconversion of vaccenic acid to conjugated linoleic acid in humans. Am. J. Clin. Nutr. 2002, 76, 504-510. [CrossRef]

36. Rist, L.; Mueller, A.; Barthel, C.; Snijders, B.; Jansen, M.; Simões-Wüst, A.P.; Huber, M.; Kummeling, I.; von Mandach, U.; Steinhart, H.; et al. Influence of organic diet on the amount of conjugated linoleic acids in breast milk of lactating women in the Netherlands. Br. J. Nutr. 2007, 97, 735-743. [CrossRef]

37. Daud, A.Z.; Mohd-Esa, N.; Azlan, A.; Chan, Y.M. The trans fatty acid content in human milk and its association with maternal diet among lactating mothers in Malaysia. Asia Pac. J. Clin. Nutr. 2013, 22, 431-442. [PubMed]

38. Ribeiro, M.; Balcao, V.; Guimaraes, H.; Rocha, G.; Moutinho, C.; Matos, C.; Almeida, C.; Casal, S.; Guerra, A. Fatty Acid Profile of Human Milk of Portuguese Lactating Women: Prospective Study from the 1st to the 16th Week of Lactation. Ann. Nutr. Metab. 2008, 53, 50-56. [CrossRef] [PubMed]

39. Sala-Vila, A.; Castellote, A.I.; Rodriguez-Palmero, M.; Campoy, C.; López-Sabater, M.C. Lipid composition in human breast milk from Granada (Spain): Changes during lactation. Nutrition 2005, 21, 467-473. [CrossRef] [PubMed]

40. Importancia del Ácido Gamma-Linolénico en la Salud y en la Nutrición. Available online: https://w3.ual.es/ $\sim\{\}$ jlguil/gamma.htm (accessed on 15 October 2019).

41. Fu, Y.; Liu, X.; Zhou, B.; Jiang, A.C.; Chai, L. An updated review of worldwide levels of docosahexaenoic and arachidonic acid in human breast milk by region. Public Health Nutr. 2016, 19, 2675-2687. [CrossRef] [PubMed]

42. Brenna, J.T.; Varamini, B.; Jensen, R.G.; Diersen-Schade, D.A.; Boettcher, J.A.; Arterburn, L.M. Docosahexaenoic and arachidonic acid concentrations in human breast milk worldwide. Am. J. Clin. Nutr. 2007, 85, 1457-1464. [CrossRef] [PubMed]

43. FAO. Standard for infant formula and formulas for special medical purposes intended for infants. Codex Aliment. 2016, 72-1981, 1-21.

44. Rueda, A.; Seiquer, I.; Olalla, M.; Giménez, R.; Lara, L.; Cabrera-Vique, C. Characterization of fatty acid profile of argan oil and other edible vegetable oils by gas chromatography and discriminant analysis. J. Chem. 2014, 2014. [CrossRef]

(C) 2019 by the authors. Licensee MDPI, Basel, Switzerland. This article is an open access article distributed under the terms and conditions of the Creative Commons Attribution (CC BY) license (http://creativecommons.org/licenses/by/4.0/). 